\title{
The efficacy of local anesthetic application to pericranial muscles in the treatment of episodic tension type headache in elderly population
}

\author{
Bilgin Öztürk ${ }^{1}$, Akçay Övünç Özön ${ }^{2}$ \\ (1) University of Health Sciences, Gulhane Training and Research Hospital, Department of Neurology, Ankara, Turkey \\ (2) Istinye University, Liv Hospital, Department of Neurology, Ankara, Turkey
}

Date submitted:

May 17, 2019

Date accepted:

Aug 05, 2019

Online publication date:

September 15, 2019

\section{Corresponding Author: \\ Bilgin Öztürk \\ University of Health Sciences, Gulhane Training and Research Hospital, Department of Neurology, Ankara, Turkey drbilgin@gmail.com}

Keywords: Tension type headache, Episodic tension type headache, Lidocaine, Treatment.

\begin{abstract}
Aims:Tension Type Headache (TTH), which is one of the primary headaches, has episodic and chronic types. Peripheral and central nociceptive mechanisms plays a role in the pathogenesis of TTH. In the acute treatment of TTH, simple and combined analgesics, and antidepressants are used for preventive therapy, but new therapy options are needed. The aim of this study is to determine the therapeutic efficacy of local lidocaine injections to pericranial muscles in elderly patients with Episodic Tension Type Headache.
\end{abstract}

Methods:Twenty-five patients with a diagnosis of episodic TTH over the age of 65 were included in the study. The patients received local $0.5 \%$ of lidocaine injections once a week for 4 sessions. Each patient received two injections to frontal muscles, temporal muscles, masseter muscles, sternocloidomastoid muscles, semispinalis capitis muscles, splenius capitis muscles and trapezius muscles bilaterally. The painful days, pain intensity (VAS-Visual Analog Scale) and the number of analgesics they had used were compared.

Results:The median age of the patients were 67 (min-max: 65-80) years. The number of painful days, pain intensity (VAS -Visual Analogue Scale) and the number of analgesics they had used after injection were statistically significantly lower $(p<0.05)$.

Conclusions:Local lidocaine injections into the pericranial muscles can be used as an effective method in the treatment of episodic TTH in the elderly.

\section{Introduction}

Headache is one of the most frequent reasons for referral to the physician at each stage as the main symptom and accompanying symptom. Primary headaches, which are usually in adulthood, have been classified into four subgroups: migraine, tension-type headache (TTH), trigeminal autonomic cephalgias and other primary headache disorders. Tension type headaches may be associated with a secondary headache as well as with another primary headache. Headache was named as, muscle contraction headache, psychomyogenic headache, stress headache, ordinary headache, essential headache, idiopathic headache and psychogenic headache in the past $(1,2)$. The annual prevalence of episodic TTH was $38.3 \%$ in community-based studies, whereas the annual prevalence of chronic TTH was $2.2 \%$ (3). The prevalence of TTH is most common in the $2 \mathrm{nd}$ and 5 th decades. Between 30 and 40 years of age is the peak $(4,5)$.

Those who have tension type headache may have a doctor's approach. As the number of painful days increases, referral to a doctor becomes more frequent. In terms of accompanying diseases; depressive disorder was found in $68.3 \%$ and anxiety disorder in $19.3 \%$ of patients with TTH. It is important to inform the patient, to record a headache diary for 4 weeks, as well as the treatment of pain and comorbid conditions (2). It is still controversial whether the pain is caused by peripheral mechanisms or from central nervous system $(6,7)$. The hypothesis suggested that nociceptive stimuli from the pericranial myofascial tissues lead to sensitization at the level of the upper cervical spinal posterior root trigeminal nucleus, and secondary to pain modulation by sensitization of supraspinal neurons such as the thalamus or somatosensory cortex (8).

There are two types of treatment modalities, both for acute or chronic TTH. The aim for the treatment of acute attack is to stop or reduce any attack; and for this analgesics are used alone or in combination (caffeine, codeine). In prophylactic treatment, antidepressant and anticonvulsant drugs are recommended as the first step and used in the treatment of chronic and episodic TTH. The side effect profile of these drugs is very wide. Certain studies have shown the efficacy of melatonin therapy in some studies, that should be considered in the presence of potentially dangerous drug interactions. Non-pharmacological treat- 


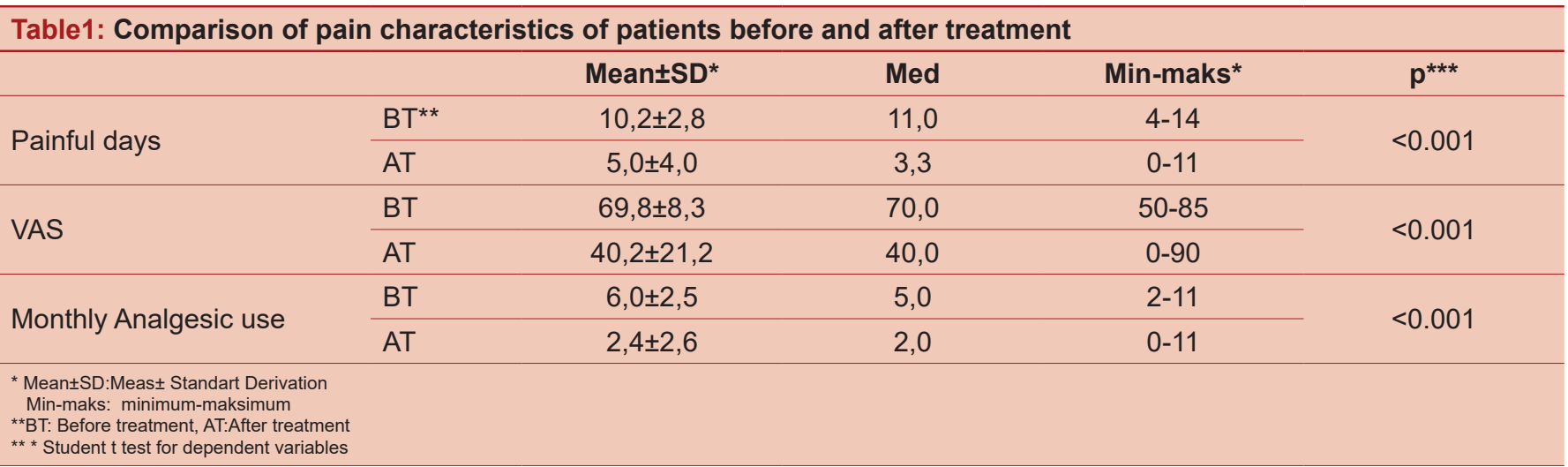

ment modalities have also been used in the treatment of TTH. Although the side effects of non-pharmacological treatment methods such as hypnosis, acupuncture, cognitive behavioral therapy and physical treatment methods are less, more scientific data are needed on their efficacy $(2,9)$.

In recent years, local anesthetic injections to pericranial muscles in the treatment of TTH have been used $(10,11)$. Some randomized, placebo-controlled studies have reported that TTH patients respond well to local anesthetic injections to pericranial muscles and are effective in the treatment (11). The aim of this study was to describe the efficacy of local lidocaine injections in the elderly with episodic TTH.

\section{Methods}

Elderly patients diagnosed with episodic TTH were included in the study. Patients with normal physical examination and neurological examinations, who were older than 65 years, with a history of at least 6 months of headaches less than 15 days per month, were included to the study. Patients with different headache types other than episodic TTH were excluded from the study. This study was approved by the local ethics committee (April 2019-19/154).

Patients that underwent to BoNT-A treatment, patients with a history of malignancy, patients underwent to cervical and cranial surgery, patients receiving non-pharmacological treatment within 6 months prior to the treatment, patients with major psychiatric disorders, patients receiving antipsychotic, antidepressant and antiepileptic drugs within 3 months of treatment were excluded.

In this retrospective study, we have evaluated the results of patients with episodic TTH who were injected with local anesthetics with the same procedure. Twenty-five patients were included in the study according to the criteria of inclusion and exclusion. Pain scores, pain intensity, visual analogue scale (VAS) and the number of analgesics used were recorded.

All patients were injected with the same procedure and procedure was done by only one neurologist. $0.5 \%$ lidocaine injections were performed to the patients once a week for one month. Each of the patients underwent bilateral injection to the frontal muscles, temporal muscles, masseter muscles, sternocloidomastoid muscles, semispinalis capitis muscles, splenius capitis muscles and trapezius muscles. For each muscle $2 \mathrm{ml}$ lidocaine was injected. The patients were evaluated 3 months after the treatment and the number of painful days, pain intensity (VAS -Visual Analogue Scale) and the number of analgesics used were recorded.
In the evaluation of descriptive statistical data, the mean \pm standard deviation, median and minimum-maximum values were used for discrete data. Student T-test was used for the dependent variables. The difference between the groups was considered as $p<0.05$ significant. SPSS 22.0 package program was used for statistical analysis.

\section{Results}

The study included 25 patients over 65 years of age with episodic tension type headache. The median values of the patients were 67 (min-max: 65-80) years. According to gender, there were 6 males (24\%) and 19 females (76\%). No statistically significant difference was found between the average age of the sexes $(p=0.77)$. The median number of painful days before the application of local anesthetics to the pericranial muscles was 11.0 and had decreased to 3.3 after the treatment. After 3 months of application, a statistically significant decrease was found in terms of the number of painful days $(p<0.001)$ (Figure1). The median VAS score for pain severity of the patients was 70.0, which had decreased to 40 after treatment. A significant decrease in VAS values was also found in terms of the severity of pain $(p<0.001)$ (Figure 2$)$. Before treatment, the median value of analgesic usage was 5 in the first month, and it had decreased to 2 after treatment. This result was statistically significant $(p<0.001)$ (Figure3). Results before and after treatment are shown in the Table1.

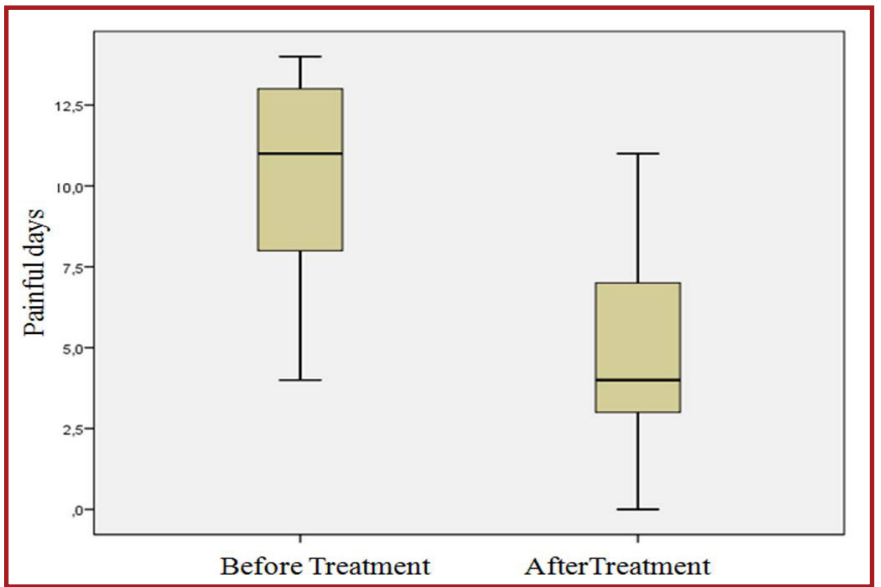

Figure 1. Comparison of painful days before and after treatment

\section{Discussion}

In this study, the efficacy of the injections of local lidocaine injections to the same pericranial muscles with standard doses 


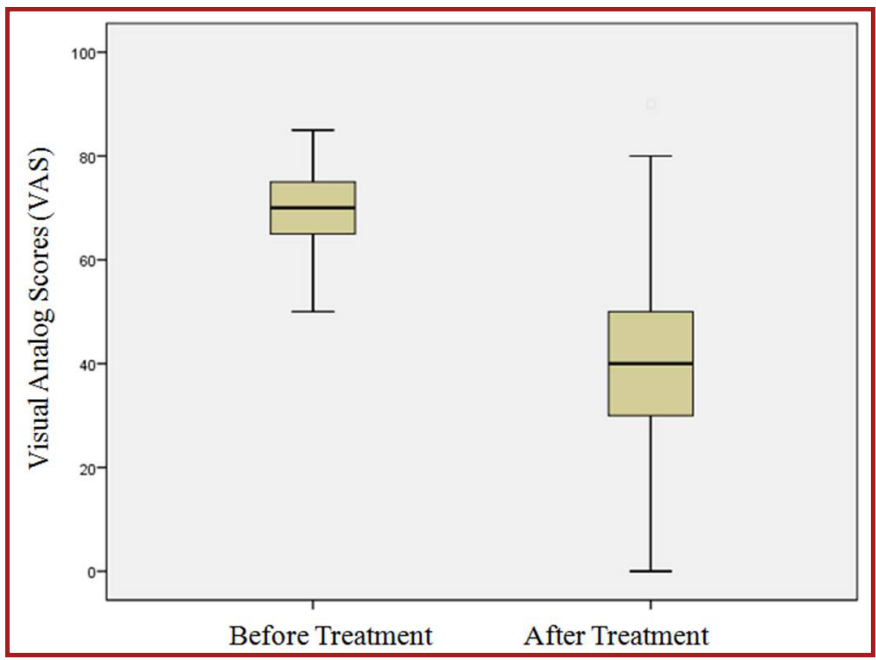

Figure 2. Comparison of VAS values before and after treatment

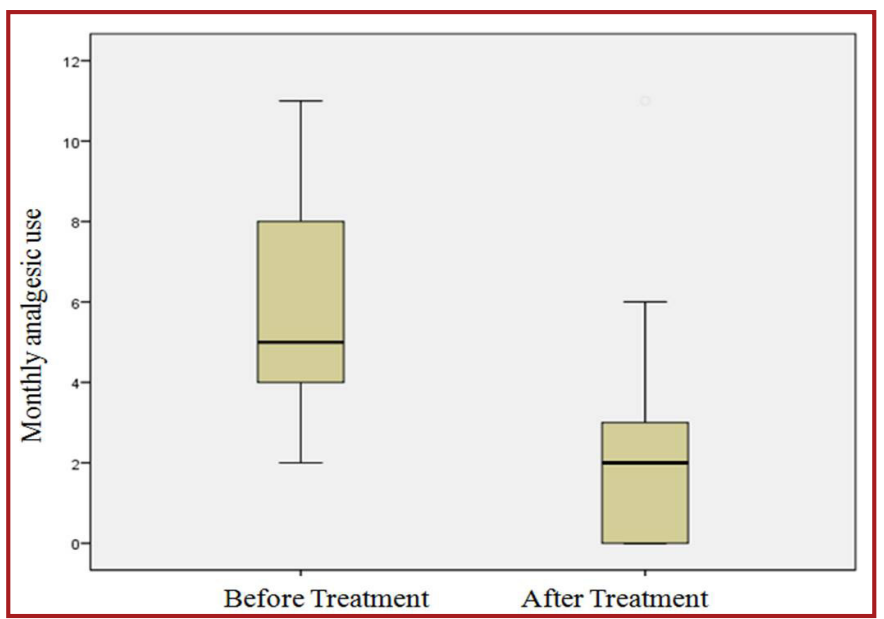

Figure 3. Comparison of analgesic use numbers of patients before and after treatment

in elderly patients with episodic TTH were investigated retrospectively. In the elderly population, 4 local lidocaine injections were administered once a week for episodic TTH patients. At the third month, data were evaluated after the applications. Local lidocaine injections were found to be effective in reducing the frequency and severity of attacks in episodic TTH after treatment. In addition, the number of analgesics taken by the elderly population for the acute treatment of episodic TTH had decreased after local lidocaine injections.

It is not clear that peripheral mechanisms or central mechanisms plays a role in the pathogenesis of TTH. However, both peripheral and central mechanisms are thought to be responsible for the pathogenesis of TTH $(4,5)$. It is possible to control the central nervous system and control the pain. The fact that pain control is provided by rearrangement of peripheral pathways with Lidocaine injection, also supports this view. Local anesthetics are drugs that reversibly block impulse conductions in nerve fibers at appropriate concentrations. The ability to produce spontaneous discharge is inhibited by local anesthetics in impulse producing cells (12).

Recent studies have focused on the importance of trigger points in many painful conditions such as primary headaches such as TTH, migraine, myofascial pain syndrome, and treatment strategies for these points have been tried to be developed. Trigger points can mediate the sensitization of trigeminal caudal nucleus neurons that receive inputs from the pericranial muscles and the determination of pain severity $(4,13)$. The main problem in TTH is the sensitization of central intermediate roads due to prolonged nociceptive inputs associated with allergenic substances from pericranial myofascial tissues $(13,14)$.

The possible mechanisms of the positive effect obtained by the local anesthetic injections to the pericranial muscles can be explained by the reduction of mediators that cause algae in these regions, the desensitization of free nerve endings and, consequently, the suppression of the prolonged pain perception process in the periphery. It is possible to increase these effects with repetitive trigger point injections and to have a long-term response. In our study, local lidocaine administration was performed as 4 sessions because of increasing the effectiveness by suppressing prolonged pain perception.

Karadaş et al. (11) performed five sessions of local lidocaine injection into the pericranial muscles of patients with episodic TTH. In another study in patients with chronic TTH; local lidocaine injection was performed to pericranial muscles, trigeminal nerve exit points and superior cervical ganglion. The number of painful days of patients, pain severity, within a month with the decrease in the number of analgesics used; Hamilton depression and anxiety scores improved (15). However, the elderly population was not evaluated in these studies. Combined applications were also performed in the chronic TTH group.

There may be transition between TTH subgroups. In particular, patients with episodic TTH are at risk for chronic TTH. Analgesic overuse is the most important factor in the conversion of episodic TTH into chronic TTH (4). In this regard, control of patients with episodic TTH without transforming into chronic TTH is important in terms of both patient comfort and treatment cost. In our study, peripheral prolonged and increased pain inputs were controlled by repeated local lidocaine injections.

As a result of our study, local lidocaine injections of recurrent pericranial muscles of the elderly with episodic TTH was found to be an effective treatment method. According to the results, it was shown that it decreased analgesic use. In addition, during the injections of lidocaine, no serious side effects were observed that would result in termination of the study. This demonstrates the reliability and tolerability of local lidocaine treatments in the preventive treatment of episodic TTH. The most important limitation of this study is the lack of a control group.

The effects of local lidocaine and pericranial muscle injections were found to be different in the treatment of tension headache. These differences; The presence of different types of headaches associated with TTH may be caused by factors such as local anesthetic dose, pharmacological combination, study design, application method and number of applications. Prospective, randomized, double-blind, placebo-controlled, and long-follow-up studies including multiple administration, different doses and different administration methods are needed to fully demonstrate the efficacy and strength of local anesthetic injections in pericranial muscles in the elderly population.

Acknowledgment: BO conducted the study, made literature search, edited and submitted the manuscript. AOO collected data from patient files, made statistical analyses, and wrote the manuscript.

Conflict of interest: The authors declared they do not have anything to disclose regarding conflict of interest with respect to this manuscript. 


\section{References}

1. Özge A, Karadaş Ö. Baş ağrısı sınıflamaları Turkiye Klinikleri J Neurol-Special Topics 2018;11(1):1-14.

2. Selçuki D, Kısabay Ak A. Gerilim Tipi Baş Ağrısı Tipleri, Tanı ve Tedavisi Turkiye Klinikleri J Neurol-Special Topics 2018;11(1):43-65.

3. Schwartz BS, Stewart WF, Simon D, Lipton RB. Epidemiology of Tension-Type Headache. JAMA. 1998;279:381383.

4. Silberstein SD, Lipton RB, Goadsby PJ. Tension-Type Headache: Diagnosis and treatment. In Clinical Practice of Headache. (2nd ed) Oxford, Martin Dunitz, 2002:113128.

5. Lipton RB, Hamelsky SW. Epidemiology and impact of headache. In: Silberstein SD, Lipton RB, Dalessio DJ, eds. Wolff's Headache and Other Head Pain. 7th ed. New York, Ny. Oxford University Press; 2001: 85-107.

6. Olesen J, Langemark M. Mechanisms of tension headache. A speculativehypothesis. In: Olesen J, Edvinsson $\mathrm{L}$, eds. Basic mechanisms of headache. Amsterdam:Elsevier, 1988:457-461.

7. Jensen K. Quantification of tenderness by palpitation and use of pressure algometers. In: Fricton JR, Awad E, eds. Adv Pain Res Ther, Vol.17. New York: Raven Press, 1990:165-181.

8. Bendtsen L, Schoenen J. Synthesis of tension-type headache mechanisms. In: Olesen J, Goadsby PJ, Ramadan N, et al., editors. The Headaches, 3rd edn. Philadelphia: Lippincott Williams \& Wilkins; 2005. pp. 677681.
9. Karadaş O, Sütçigil L, Odabaşı Z. Kronik Gerilim Tipi Baş Ağrısı Tanısı Alan Hastalarda Melatonin Tedavisinin Etkinliği ile Anksiyete ve Depresyon Üzerine Etkisi J.Neurol.Sci.[Turk] 2013;30(1) 34; 116-123.

10. Karadaş $O$, Babacan A, Gül LH, Ipekdal IH, Türk Börü $U$. [The role of pericranial muscles in the succesful management of episodic tension type headache]. Agri. 2012;24(4):153-158.

11. Karadaş Ö, Gül HL, Inan LE. Lidocaine injection of pericranial myofascial trigger points in the treatment of frequent episodic tension-type headache. J Headache Pain. 2013 May 22;14:44.

12. William A. Catterall and Kenneth Mackie: local anesthetics, in Brunton LL, lazo JS, parker kKL(eds): Goodman \& Gilman's the pharmacological basis of therapeutics, ed 11. Texas, The Mcgraw-hill Mompanies Inc, 2006, pp 241-251.

13. Bendsten L. Central sensitization in tension type headache-possible pathophysiological mechanisms. Cephalalgia 2000;20:486-508.

14. Jensen R. Peripheral and central mechanisms in tension-type headache: an update. Cephalalgia. 2003;23(Suppl. 1):49-52.

15. Karadaş Ö, Inan LE, Ulaş Ü, Odabaşi Z. Efficacy of local lidocaine application on anxiety and depression and its curative effect on patients with chronic tension-type headache. Eur Neurol. 2013;70(1-2):95-101. 